\title{
Identification of Ammonia in Gas Emanated from Human Skin and Its Correlation with That in Blood
}

\author{
Kazutoshi Nose,* Tatsuo Mizuno,* Norio Yamane,** Takaharu Kondo, ${ }^{* *}$ Hajime Ohtani,* \\ Shuki ARAKI,* and Takao TsudA***ं
}

*Department of Materials Science and Engineering, Graduate School of Engineering,

Nagoya Institute of Technology, Gokiso, Showa, Nagoya 466-8555, Japan

**Research Center of Health, Physical Fitness and Sports, Nagoya University,

Furo-cho, Chikusa, Nagoya 464-8601, Japan

***Pico-Device Co., Incubation Center Office 5, Nagoya Institute of Technology,

Gokiso, Showa, Nagoya 466-8555, Japan

\begin{abstract}
Identifying and measuring the ammonia gas that emanates from human skin, which we called skin gas, has been achieved using a modified gas chromatographic system with a nitrogen-selective detector (flame-thermoionic detector: FTD). The skin gas is collected with a home-made sampling probe or bag, which is used to cover the skin surface of a subject's wrist, or a finger, for $5 \mathrm{~min}$. It was proved that ammonia was present in skin gas for healthy persons and patients with hepatic disease. The average amounts of ammonia were $1.7 \pm 0.4$ and $2.7 \pm 0.8 \mathrm{ng} / \mathrm{cm}^{2}$; furthermore, there was a significant difference between them $(p<0.05)$. In addition, the ammonia levels present in skin gas were correlated with that in blood $(r=0.64, p<0.05)$.
\end{abstract}

(Received July 28, 2005; Accepted October 5, 2005)

\section{Introduction}

Non-invasive analytical tests are important in human healthcare. ${ }^{1-7}$ Breath, ${ }^{1,2,8}$ sweat, ${ }^{5-7}$ and saliva ${ }^{9}$ are used for such tests. Caffeine, ${ }^{5}$ lactate, ${ }^{6}$ and alkali metals ${ }^{7}$ can be identified in sweat or in inner-body fluids under the skin. In addition, gases emanating from human skin, which we call skin gas, can be collected with minimum stress for the subject. ${ }^{1-4}$ Since the components in skin gas are generally in quite low levels, it is necessary to detect them with a highly sensitive detector. We have already identified hydrogen,,${ }^{1,2}$ acetone,,${ }^{1,4}$ and methane ${ }^{3}$ in skin gas with a modified gas chromatographic system. ${ }^{10}$ It is clear that the amounts of hydrogen and acetone in skin gas correlate to that in the subject's breath. ${ }^{1,2}$ Furthermore, there is a well-established relationship between the amounts of acetone contained in skin gas and the concentrations of 3hydroxybutyrate in blood. ${ }^{4}$

It is known that the ammonia levels in the blood of patients with liver cirrhosis is higher than that in healthy persons, especially in those patients suffering from hepatic encephalopathy. Therefore, ammonia levels are used for the diagnosis of hepatic encephalopathy in cirrhotic patients. ${ }^{11}$

The present study presents the first report that a proof of the existence of ammonia in gas emanating from human skin (skin gas) is provided by using a modified gas chromatographic system with a nitrogen selective flame-thermoionic detector (FTD). Furthermore, we examined two fundamental features of ammonia present in skin gas as follows. One of the features

$\uparrow$ To whom correspondence should be addressed.

E-mail: tsuda@pico-device.co.jp was whether there was a significant difference between the ammonia levels in the skin gas of healthy subjects and in that of subjects with hepatic disease. The other was the relationship between the ammonia amounts in skin gas and that in blood.

\section{Experimental}

\section{Materials}

A gas sampling probe made from polytetrafluoroethylene was used to collect gas emanating from human skin. The probe was $33 \mathrm{~mm}$ square and its height was $11 \mathrm{~mm}$. The probe was carved with a whirlpool pattern on its base (depth, $1 \mathrm{~mm}$; width, $3 \mathrm{~mm}$; total length, $191 \mathrm{~mm}$ ), and it covered $522 \mathrm{~mm}^{2}$ of skin surface and had a total volume capacity of $573 \mathrm{~mm}^{3}$ for the gas route. ${ }^{1}$ The ammonia aqueous solution was commercially obtained from Wako Pure Chemical Industries, Ltd. (Osaka, Japan). A calibration gas was prepared from diluted solutions.

\section{Sampling procedure}

Sampling skin gas - method I. Two different methods (I and II) were used to collect skin gas. Method I was suitable for identifying ammonia in skin gas and for investigating the relationship between the amount of ammonia in skin gas and its concentration in blood. Skin gas was collected by covering the skin of the left wrist with a polytetrafluoroethylene probe (shown in Fig. 1). This sampling procedure was conducted in the following way: first, the part of the wrist required for the sampling was washed with running tap water for $30 \mathrm{~s}$ and sprayed with distilled water for $5 \mathrm{~s}$. The wrist was then wiped off with paper (Kimwiper S-200, Kimberly-Clark Co., supplied by Kulesia, Tokyo) and left to dry naturally for $5 \mathrm{~min}$. Second, 


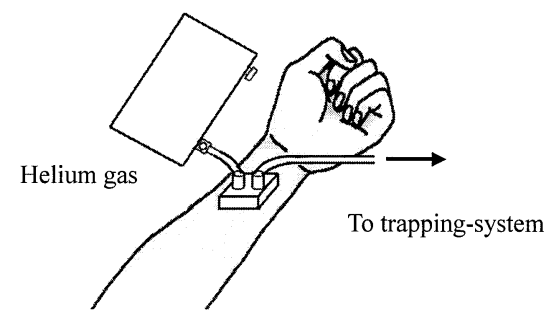

Fig. 1 Schematic diagram of skin gas sampling using method I.

the probe was held against the wrist and helium gas was passed through the probe for $1 \mathrm{~min}$ at a flow rate of $50 \mathrm{~mL} / \mathrm{min}$ to substitute for the original gas in the probe. Then, skin gas from the wrist was collected for $5 \mathrm{~min}$ using the probe. At other times, a blank gas was collected using the same procedure, but the skin surface was substituted with a polytetrafluoroethylene sheet.

Sampling of skin gas - method II. Method II was used in a hospital to sample the skin gas of healthy subjects and subjects with hepatic disease. This sampling procedure was described in a previous report. ${ }^{4}$ The procedure is briefly described as follows: skin gas was collected by holding a subject's finger inside a handy gas collector (made from a perfluorinated ethylene-propylene copolymer bag and filled with $50 \mathrm{~mL}$ room air) for $5 \mathrm{~min}$. The collected skin gas was transferred in a poly(vinyl fluoride) bag and analyzed using a modified gas chromatographic system.

Blood sampling. Blood samples were collected in the following way. First, the tip of a finger was thoroughly washed with distilled water for $10 \mathrm{~s}$, and soaked in a $70 \%$ ethanol solution containing $0.1 \%$ chlorhexidine gluconate (Hibitane ${ }^{\circledR}$ gluconate solution, Sumitomo-ICI pharma, Co.) for $1 \mathrm{~min}$. The tip of a finger was thoroughly wiped with paper (Kimwiper) soaked in an ethanol solution. After it being dried, the finger was punctured with a lancet, and a $20 \mu \mathrm{l}$ sample of blood was taken from a capillary.

The blood samples were treated carefully to avoid any contamination. Because ammonia also exists in sweat ${ }^{12}$ and in the atmosphere, the value of ammonia in the blood will give a higher value when these are mixed with blood. This problem was solved by thoroughly washing the finger as outlined above and taking the blood sample as quickly as possible.

\section{Procedure for trapping a gas sample}

The identification of ammonia present in skin gas can be achieved using a modified gas chromatographic system. ${ }^{10}$ The trapping procedure for skin gas collection was as follows: a skin gas sample was automatically introduced into a stainless-steel tubing (inner diameter $0.8 \mathrm{~mm}, 10 \mathrm{~cm}$ long, and inner volume $0.05 \mathrm{ml}$ ) for $10 \mathrm{~s}$ at a flow rate of $50 \mathrm{~mL} / \mathrm{min}$. In this process, the stainless-steel tubing was cooled with liquid nitrogen at $-196^{\circ} \mathrm{C}$. After the switching valve of the system was rotated, the trap tubing was heated to $80^{\circ} \mathrm{C}$ and the trapped components in skin gas sample were subjected to rapid thermal desorption. Accordingly, the components were introduced into the gas chromatograph, and analyzed.

\section{Analytical conditions}

Measurement of ammonia levels in gas samples. The levels of ammonia present in the gas samples were detected with a gas chromatograph (GC-14B, FTD, Shimadzu, Kyoto). The GC separation conditions for determining the ammonia levels in skin gas were as follows: a packed column (Chromosorb 103 , $3.2 \mathrm{~mm}$ inner diameter and $1.6 \mathrm{~m}$ long); the injection and detection port temperature, $150^{\circ} \mathrm{C}$; and the column temperature, $80^{\circ} \mathrm{C}$. Under these conditions, the ammonia's retention time was $1.8 \mathrm{~min}$, and the GC analysis took $4 \mathrm{~min}$, as shown in Fig. 2 .

Ammonia was detected with a flame-thermoionic detector, which is a type of nitrogen-selective detector. Its sensitivity was enhanced using a carrier gas that contained a small amount of methane (ca. $0.989 \%$ )

Measuring the concentration of ammonia in blood. Amicheck and PocketChem BA (Arklay, Kyoto) were used to measure the concentration of ammonia in blood samples. The ammonia present in the blood sample reacted with the bromcresolgreen in Amicheck, and its concentration was determined by measuring the absorbance of the reaction product with PocketChem BA. In this method, the whole blood sample was used for the measurement.

\section{Subjects}

A total of 28 healthy volunteers and 24 volunteers diagnosed with hepatic disease were involved in the experiment. Informed-consent was obtained from each subject. This study was approved by the ethics panel of the Research Center of Health, Physical Fitness and Sports, Nagoya University (\#0114). The ages of the healthy subjects and the subjects with hepatic disease were $71 \pm 15$ and $64 \pm 16$ [mean \pm SD], respectively.

\section{Protocols}

Comparison of the ammonia amounts in skin gas in healthy subjects and that in subjects with hepatic disease. The subject samples of 24 healthy subjects and 24 patients with hepatic disease consisted of inpatients and outpatients. All skin gas samples were collected from subjects before breakfast on the morning after their fast, to avoid food intake influencing the result.

Relationship between the ammonia amounts in skin gas and that in blood. To avoid food intake influencing the results, the four healthy subjects fasted for $12 \mathrm{~h}$ before the experiment. The skin gas and blood sampling was performed at the same time every 30 min after the intake of a supplement that contained $17 \mathrm{~g}$ of protein. For comparison, a sample was also taken before the intake of protein.

\section{Calibrations}

The standard original 25\% ammonia aqueous solution was diluted with distilled water to $1.6,3.3,5.1,6.6$ and $8.3 \mathrm{mmol} / \mathrm{L}$ ammonia aqueous solutions. The $20 \mu \mathrm{L}$ portions of these diluted ammonia solutions were introduced into the individual poly(vinyl fluoride) bags with $1 \mathrm{~L}$ of helium gas. The concentrations of the prepared calibration gases were $0.74,1.48$, $2.28,2.96$ and $3.70 \mathrm{ppm}$, respectively. The calibration curve had a good first-order linear relationship, with a correlation factor of 0.99 .

\section{Calculating total amount of ammonia in skin gas}

The total amount of the ammonia in skin gas, $w$ (ng), was calculated by

$$
w=\frac{m V M}{22.4} .
$$

The terms $m$ (ppm), $V(\mathrm{~mL})$, and $M(\mathrm{~g} / \mathrm{mol})$, respectively, indicate the concentration of ammonia in skin gas, the gas volume introduced in the trapping-system, and the mol weight 

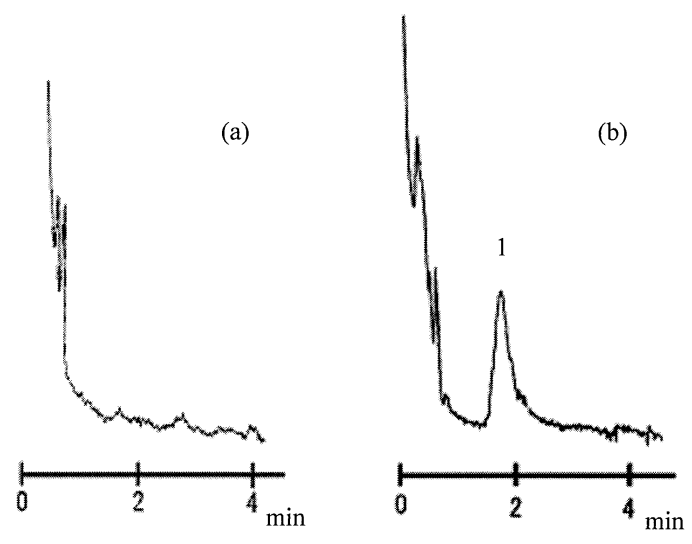

Fig. 2 Typical chromatograms of (a) blank gas (b) and skin gas. Peak: (1) ammonia.

of ammonia (in this study: $V=8.3, M=17.03$ ). In Eq. (1), the number 22.4 indicates the conversion factor based on the ideal gas law.

\section{Statistical examination}

In this study, the significance test was based on the Student's $t$ test. If the $p$ value was smaller than 0.05 , i.e. $p<0.05$, we assumed that there was statistical significance.

\section{Results and Discussion}

\section{Identification of ammonia in skin gas}

Some typical chromatograms of the blank gas and skin gas that emanated from a subject's wrist, and which were collected using method I, are shown in Figs. 2-a and 2-b, observed using a nitrogen-selective detector. While no peak appeared in the chromatogram of the blank gas, there was a sharp peak in that of the skin gas. Peak 1 (Fig. 2-b) was identified chromatographically by comparing the retention time of the peak with that of the ammonia present in the standard ammonia gas. In fact, the retention time of peak 1 was perfectly consistent with that of the standard. Consequently, it was clear that the ammonia gas emanated from the skin surface.

Comparison of the amount of ammonia in skin gas for healthy subjects and that in subjects with hepatic disease

The skin gas was collected with method II from the healthy subjects and the subjects with hepatic disease. The average amounts of ammonia present in skin gas were $1.7 \pm 0.4$ and 2.7 $\pm 0.8 \mathrm{ng} / \mathrm{cm}^{2}$, respectively. Figure 3 shows the difference in the amount of ammonia among them. The results show that the ammonia was clearly present in skin gas for all of subjects, and there was a significant difference between the amounts of ammonia present in the skin gas of the healthy subjects and in those suffering hepatic disease $(p<0.05)$.

Most of the ammonia generated is converted into urea during the urea cycle in the liver and is excreted by the kidney in urine. ${ }^{16}$ The amount of ammonia in the skin gas of subjects with hepatic disease, who displayed a weak detoxication function in converting ammonia to the urea was, in general, larger than that of the healthy subjects. This result implies that the concentration of ammonia in skin gas might be used as an index of hepatic disease, including liver cirrhosis and hepatic encephalopathy.

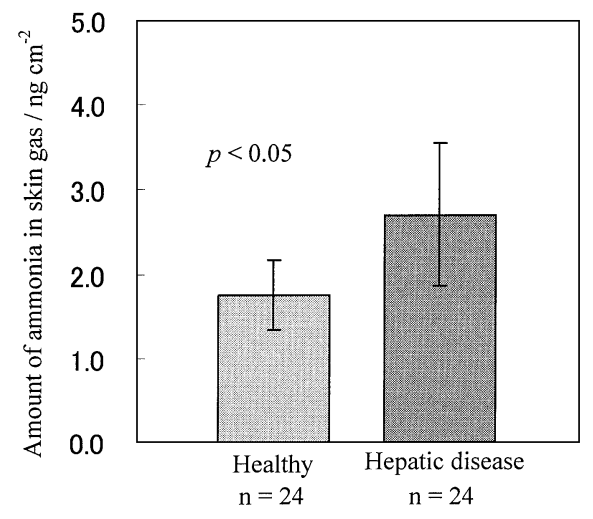

Fig. 3 Amounts of ammonia present in skin gas of healthy subjects and subjects with hepatic disease. Sampling period, $5 \mathrm{~min}$.

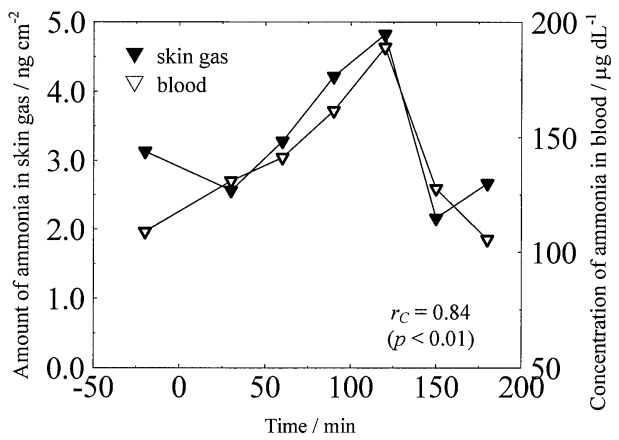

Fig. 4 Variation in the amount of ammonia present in skin gas and the concentration of ammonia in the blood sample of a healthy subject C. The $r_{\mathrm{c}}$ was the correlation factor for subject C. Sampling period, $5 \mathrm{~min}$.

\section{Variations and correlations of ammonia in skin gas and blood}

The variations of ammonia in skin gas collected with method I and blood after the subject's protein intake are shown in Fig. 4. It is clear that both the amount of ammonia in the skin gas and the concentration of ammonia in the blood increased after the protein intake, and the ammonia concentration reached the maximum level after about a 2 -h period. Similar variations were recorded in the ammonia levels in skin gas and blood samples from the other subjects. The average ammonia values in the skin gas of four of the subjects varied from $2.0 \pm 0.9$ (before the intake of protein) to $3.3 \pm 1.1 \mathrm{ng} / \mathrm{cm}^{2}$ (maximum), in blood that varied from $111 \pm 13$ to $171 \pm 21 \mu \mathrm{g} / \mathrm{dL}$, respectively. The maximum ammonia values were larger than those obtained before the intake of protein in skin gas and blood. These $p$ values were 0.06 and less than 0.01 , respectively. Ammonia in vivo is usually generated by the deamination of amino acids in the metabolic processes of the protein. ${ }^{13}$ Various amino acids, produced as the metabolite of the proteins, are converted into glutamic acid. Ammonia is then generated by the action of the glutamate dehydrogenase in the mitochondria. The amount of ammonia in the blood increases after an intake of protein ${ }^{14,15}$ due to the above process. Consequentially, the amount of ammonia in the skin gas also increased according to the results of the same process.

In addition, the relationship between the amount of ammonia present in skin gas and its concentration in the blood samples of subjects is shown in Fig. 5. The results showed that the 


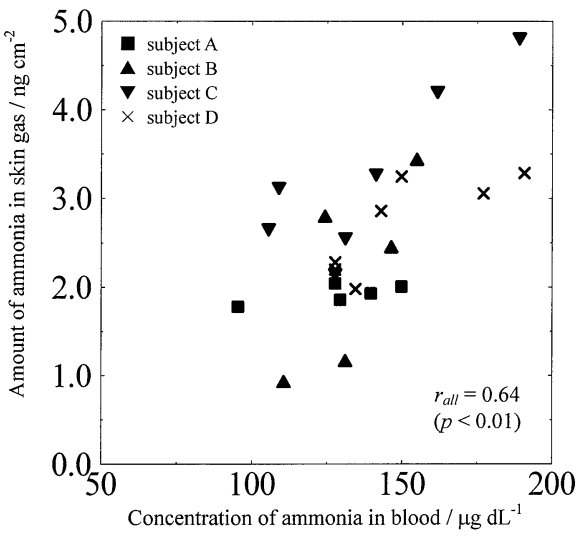

Fig. 5 Relationship between the ammonia levels present in skin gas and that in the blood samples of four healthy subjects taken at various periods. Sampling period, $5 \mathrm{~min}$.

ammonia in skin gas was correlated with that in blood, with the correlation factor $r_{\text {all }}=0.64(p<0.01)$. Let us examine the results in detail. The individual correlation factors for the subjects were $0.73,0.73,0.84$, and 0.80 , respectively.

The reason the $r_{\text {all }}$ was inferior to individual correlation factors was that the ammonia levels in skin gas differed widely among each individual in comparison to that in blood.

\section{Conclusion}

In this study, we successfully identified trace amounts of ammonia present in skin gas using a gas chromatographic system with a nitrogen-selective detector (FTD). We proved that ammonia was clearly present in gas emanated from human skin for all of the healthy volunteers and those with hepatic disease. In addition, we discovered that there was a significant difference in the ammonia levels between them. Furthermore, we found that the ammonia levels in skin gas were correlated with that in blood and that the individual correlation factors of these are better than the entire correlation factors of the four subjects. On the other hand, it is important that the skin gas can be collected easily with less stress for the people. By a further study for the features of skin gas, a diagnosis of liver disease using skin gas might be possible with continuous monitoring of the variation of ammonia levels between individuals. For example, when a home-care apparatus for testing ammonia levels becomes available for measuring the ammonia in skin gas, it will be possible to apply to this to home medical care after receiving a diagnosis at the hospital in the future. When an increase in ammonia levels is detected, a patient can then seek medical treatment.

\section{Acknowledgements}

This work was partly supported by the Promotion Program, Aichi Science and Technology Foundation. Thanks to the Chemicals Evaluation and Research Institute, Tokyo and $\mathrm{Mr}$. Hiroyuki Sugiura at Takasago Electric, Inc. for their help.

\section{References}

1. K. Naitoh, T. Tsuda, K. Nose, T. Kondo, A. Takasu, and T. Hirabayashi, Instrum. Sci. Technol., 2002, 30, 267.

2. T. Kondo, T. Tsuda, K. Nose, H. Ishiguro, T. Mitsui, K. Gao, and K. Fujiki, Am. J. Gastroenterol., 2002, 97, 1271.

3. K. Nose, Y. Nunome, T. Kondo, S. Araki, and T. Tsuda, Anal. Sci., 2005, 21, 625.

4. K. Nose, T. Kondo, S. Araki, and T. Tsuda, Bunseki Kagaku, 2005, 54, 161.

5. T. Tsuda, N. Naitoh, N. Mizuno, S. Kitagawa, and A. Ishii, Jpn. J. Perspiration Res., 2001, 8, 13.

6. S. Sugase and T. Tsuda, Bunseki Kagaku, 2002, 51, 429.

7. J. Terasawa, K. Mitsuya, A. Ishii, and T. Tsuda, Bunseki Kagaku, 2001, 50, 813.

8. C. Shimamoto, I. Hirata, and K. Katsu, Hepatogastroenterology, 2000, 47, 443.

9. I. Nakamoto, K. Morimoto, T. Takahita, and M. Toda, Environ. Health Prev. Med., 2003, 8, 95.

10. K. Naitoh, Y. Inai, and T. Hirabayashi, Anal. Chem., 2000, $72,2797$.

11. J. P. Ong, A. Aggarwal, D. Krieger, K. A. Easley, M. T. Karafa, F. V. Lente, A. C. Arroliga, and K. D. Mullen, Am. J. Med., 2003, 38, 35 .

12. D. Czarnowski, J. Gorski, J. Jozwiuk, and A. BoronKaczmarska, Eur. J. Physiol., 1992, 65, 135.

13. V. Felipo and R. F. Butterworth, Prog. Neurobiol., 2002, 67, 259.

14. O. Hamberg, K. Nielsen, and H. Vilstrup, J. Hepatol., 1992, 14, 237.

15. J. Kromhout, J. C. McClain, L. Zieve, M. W. Doizaki, and S. Gilberstadt, Am. J. Gastroenterol., 1980, 74, 507.

16. D. A. Diamond, A. Blight, C. T. Samuell, and P. G. Ransley, Brit. J. Urol., 1991, 67, 541. 